\title{
Application of infrared thermography for evaluation of patch test results
}

\author{
by B. Tomaka*, M. Szwedo** and J. Targosz* \\ * AGH University of Science and Technology, 30 Mckiewicza Av., 30-059 Krakow, Poland, tomaka@agh.edu.pl \\ ** MONIT SHM Sp. z o.o., 34 Lublanska Str., 31-476 Krakow, Poland, mszwedo@monitshm.pl
}

\begin{abstract}
Infrared thermography is not commonly used in allergological diagnostics. In our research we focus on application of infrared thermography for evaluation of patch test results, which is acknowledged as the gold standard and commonly used in contact dermatitis diagnostics. Evaluation of this tests do concern only skin surface because it depends on visual assessment thus it cannot be treated as an objective method. We develop the procedure of data acquisition for tests performed on patients, which allows to check the difference in skin temperature at regions where patch tests were incubated in comparison to the neighbouring skin regions and reference temperature.
\end{abstract}

\section{Introduction}

Infrared thermography is not commonly used in dermatological and allergological diagnostics. It is in scientist's interest to evaluate it, because as a non-invasive, non-contact and relatively cheap measurement technique may be a valuable tool for future interpretation of various medical examination results. For example Laino et al. [1] described problems with hot areas that extended beyond the borders of the patch site. They have pointed out the necessity to keep large intervals between patches, like 8 to $10 \mathrm{~cm}$. In the study of Dencheva et al. [2] they acquired 100\% matching of clinical and thermal image results for negative skin reaction and $60,7 \%$ for positive.

Few years ago the International Standard Organization has published two standards to define the use of a thermography in fever screening. The first one, IEC 80601-2-59:2008, describes particular requirements for the basic safety and essential performance of screening thermography for human febrile temperature screening, and the second one, ISO/TR 13154:2009, defines deployment, implementation and operational guidelines for identifying febrile humans using a screening thermograph [1,3,4]. However it is about fever screening it provides some guidelines for application of thermal imaging in clinical medicine. These documents dictate standards in manufacture and performance and are specific in the description of calibration procedures $[5,6]$.

Generally skin tests are used in allergological diagnosis and its principle is to attempt to provoke a small, controlled, allergic response. Allergic reactions occur when a person's immune system reacts to allergens, which are normally harmless substances in the environment. In case of patch tests this reaction results in an inflammatory response caused by a type-IV cell-mediated reaction with release of proinflammatory cytokines increase permeability of blood vessels and with typical local symptoms like redness, temperature increase and eczema with erythema, papules, vesicles and weeping. This inflammatory lesions can spread beyond the area of contact to other part of the body, often in a symmetrical pattern [7]. Allergic contact dermatitis (ACD) lesions usually appear 24-48 hours after the last exposure to the allergen and reach their peak at approximately 72-96 hours, but they may develop as early as 5 hours or as late as 7 days after exposure [8]. Patch testing is the only useful and reliable method for identification of an allergic contact dermatitis - it is acknowledged as the gold standard. For over forty years major role in the standardisation of patch testing have the International Contact Dermatitis Research Group (ICDRG) which was formed to promote the understanding of contact dermatitis [9].

In patch test, strips of tape containing small quantities of common allergens are applied onto the skin, usually on the back, for 48 hours incubation. The skin reaction is observed at regular intervals, usually after 48,72 and 96 hours [5, 9]. Evaluation of the test by a physician does concern only skin surface because it depends on visual assessment. Thus difficulties like changes in skin pigmentation, atopic skin, black race skin or delayed reaction may occur and as a result it cannot be treated as an objective method. Common problem occurring during patch test results evaluation is connected with interpretation of lesions as irritant, doubtful and weak reaction, especially at second reading, when uncertain grades are not allowed. The issue is that in case of negative grade at second reading it matters if interpretation of reaction at first reading was either doubtful (+/-) or weak (+). Wrong diagnostics extends time of final diagnosis, generates costs by necessity of another physical and causes discomfort of a patient.

In our research we focus on application of infrared thermography for evaluation of patch test results. We develop the procedure of data acquisition for tests performed on patients, which allows to check the difference in skin temperature at regions where patch tests were incubated in comparison to the neighbouring skin regions and reference temperature. 


\section{IR thermography in patch test diagnosis}

\subsection{Skin thermal parameters}

There are three main layers of skin: the epidermis, the dermis and the subcutaneous layer. The epidermis as the outer layer of the skin is directly above the capillary bed which supplies the skin with blood. The thickness of the epidermis varies on different locations of the body, from $0,05 \mathrm{~mm}$ up to $1,5 \mathrm{~mm}$. Below the epidermis is the dermis which is the middle layer of the skin and contains the capillary bed, nerves, sweat glands, etc. The subcutaneous region is the deepest layer of skin. It consists of a network of collagen and fat cells and allows pathways for the blood vessels into the dermis. It helps conserve the body's heat and protects the body from injury by acting as a shock absorber [11].

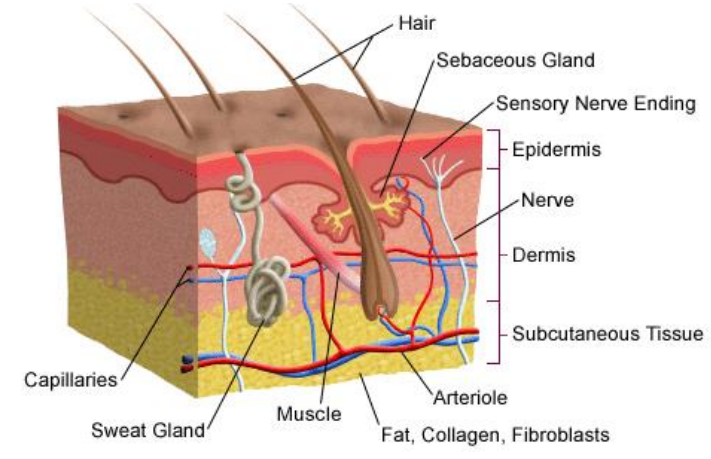

\begin{tabular}{|l|c|c|}
\hline \multicolumn{1}{|c|}{ Skin layer } & $\begin{array}{c}\text { Thermal } \\
\text { conductivity } \\
{[\mathrm{W} /(\mathrm{m} \cdot \mathrm{K})]}\end{array}$ & $\begin{array}{c}\text { Specific heat } \\
{[\mathrm{J} /(\mathrm{kg} \cdot \mathrm{K})]}\end{array}$ \\
\hline Epidermis & 0,24 & 3500 \\
\hline Dermis & 0,45 & 3300 \\
\hline $\begin{array}{l}\text { Subcutaneous } \\
\text { tissue }\end{array}$ & $0,21-0,64$ & $1400-4000$ \\
\hline
\end{tabular}

Fig. 1. The skin structure [11] and its thermal parameters [12].

For proper thermal analysis it is important that the tissue is non-homogenous medium with non-linear, temperature and time dependent thermal properties. Temperature distribution in tissues depends on few parameters, like thermal conductivity, convection heat transfer, metabolic heat production, external heat transferred to the tissue, time, tissue's geometry. Pennes [13] suggested that the rate of heat transfer between blood and tissue is proportional to the product of volumetric perfusion rate and the difference between the arterial blood temperature and the local tissue temperature. It is expressed by Eq. (1)

$$
Q_{b}=V \rho_{b} C_{b}(1-\kappa)\left(T_{a}-T\right)
$$

where: $Q_{b}\left[\mathrm{~W} / \mathrm{m}^{3}\right]$ - rate of heat transfer per unit volume of tissue, $\rho_{b}-$ density of blood, $C_{b}-$ specific heat of blood, $k-$ factor that accounts for incomplete thermal equilibrium between blood and tissue, $T_{a}$ - temperature of arterial blood, $T$ local tissue temperature.

Following Eq. (1) the thermal energy balance for perfused tissue is as shown at Eq. (2)

$$
\rho C \frac{\partial T(x, y, z, t)}{\partial t}=k \nabla^{2} T(x, y, z, t)+Q_{b}+Q_{m}
$$

where: $C$ - specific heat, $\rho$ - density of tissue, $k$ - thermal conductivity of tissue, $T(x, y, z, t)[\mathrm{K}]-$ temperature at time $t, Q_{m}$ - rate of metabolic heat production per unit volume of tissue.

According to $[14,15,16]$ the essence of the thermography is to compare temperature of the skin with positive reaction to allergen with temperature of surrounding healthy skin, so information about gradient of temperature is more important than its absolute value. Emissivity of biological tissues is within 0.85 and 0.98 . For human skin it is reported to be equal $0.98 \pm 0.01$ for wavelength range of $2-20 \mu \mathrm{m}$, it peaks around $10 \mu \mathrm{m}$, regardless of skin colour. Generally, for medical applications narrower wavelength band is used, from 8 to $12 \mu \mathrm{m}$. Maximal gradient for the strongest allergic reactions is within 2 and $3^{\circ} \mathrm{C}$ (accuracy of measurement depends on parameters of used camera).

\subsection{Standard requirements for medical thermography}

Infrared camera should be mounted perpendicularly to the examined surface, emissivity should be settled at 0,98 and sensitivity and accuracy should be defined [14]. Consulting room has to fulfil the following requirements: ambient temperature within $20-23^{\circ} \mathrm{C}$, air humidity within $45-55 \%$, lack of external heat and radiation sources, no draught and enough space. Those parameters have to be written down in the test record form.

In case of thermal imaging, very important part of examination is patient's preparation. It is not allowed to apply substances, medications or drugs and to practise physical exercises even for a few days before test application. Before readings patient should come calmly and for 15 to 30 minutes stay in rest at waiting room. Then acclimatization in consulting room with undressed parts of the body, which will be studied, takes from 5 to 30 minutes. Such rules have to provide better accuracy and repeatability of the measurements. [6, 17] 


\subsection{1/qirt.2016.136}

\subsection{Proposed measurement procedure for evaluation of patch test results}

In reference to results of our primary tests described in [18], where results obtained with use of infrared thermography were valuable and promising but not unambiguous, we are developing the procedure of data acquisition. Although measurement conditions were almost the same, it was not possible to compare obtained thermographs so we implemented a reference temperature module for our measurement stand.

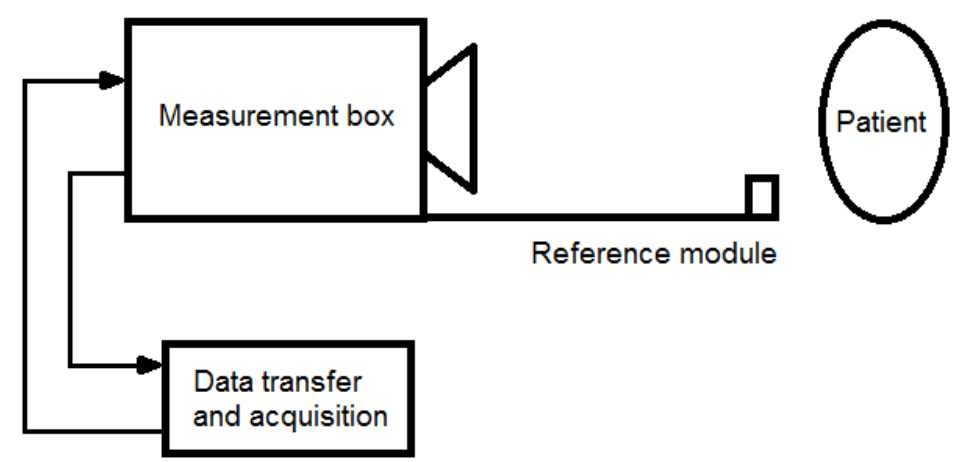

Fig. 2. Schematic of measurement unit.

To obtain better diagnostic results with reduced patient discomfort during medical examination we are designing dedicated measurement stand. Schematic of developed prototype system for patch tests evaluation is presented at Fig. 2. It consist of measurement box with reference module and mobile computer for image acquisition and control of measurement parameters. Inside measurement box we have placed: infrared camera FLIR A300, industrial camera PhotonFocus, system of lights and power supply unit with programmable digital microcontroller ATmega16.

FLIR A300 is equipped in a standard uncooled microbolometer FPA with resolution 320x240 pixels and image frequency $30 \mathrm{~Hz}$. Vision camera, made in CMOS technology, has resolution 2048x1088 pixels and wide spectral range. Application of this two types of cameras will enable image correlation between the visual and thermal images. System of lights is required for vision camera and in that usage it is a set of lights with different wavelength to improve detection of lesions. Phase lock of cameras and synchronization with lights is controlled by programmable digital microcontroller ATmega16 in correlation with ThermoAnalysis software.

a)

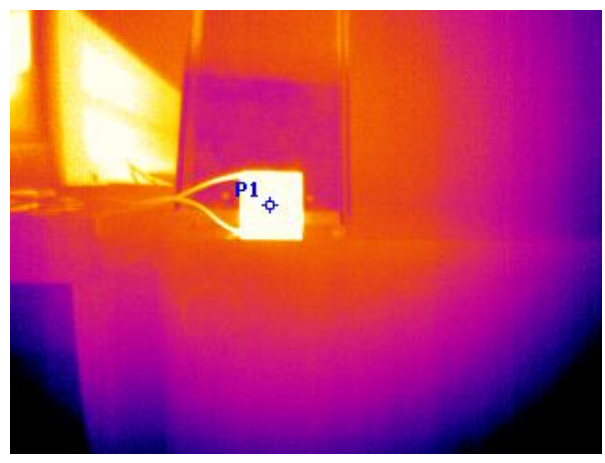

b)

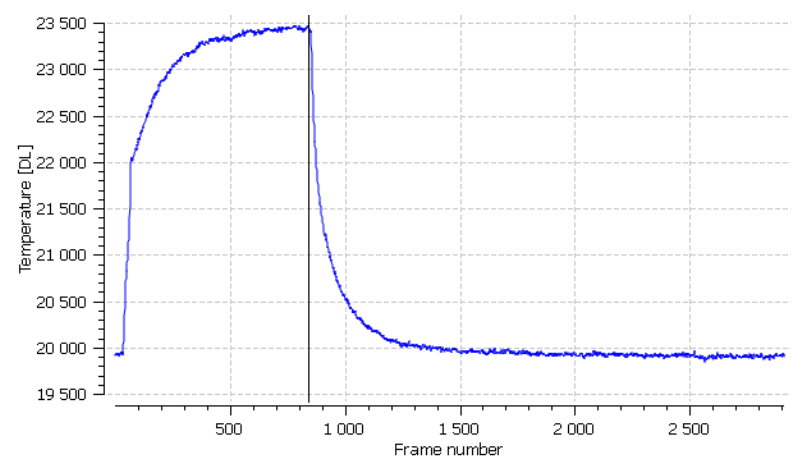

c)

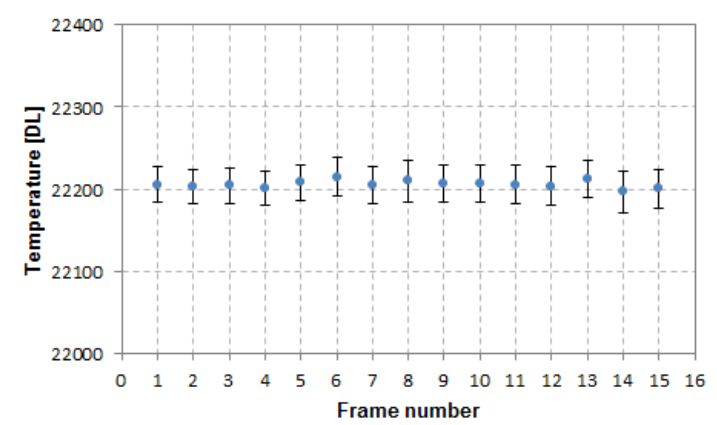

Fig. 3. Heating function of Peltier module at point $P 1$ : a) thermograph with marked point $P 1$, b) heating function, c) standard deviation for a part of the function. 


\subsection{1/qirt.2016.136}

As a reference module we used a Peltier module $(12 \mathrm{~V}, 60 \mathrm{~W})$ settled in our measurement stand in the way that it is positioned near examined object and is in the IR camera field of view. To be sure that our results will be valuable we have checked the heating function of that module with $2 \mathrm{~V}$ power supply. We used FLIR A300 camera for that purpose with frame rate set on $3 \mathrm{~Hz}$ and make measurements 16 times. Received results are presented at Fig. 3 where standard uncertainty is shown for a fragment of the function including 15 fames at its horizontal maximum stable part.

Used in tests Peltier modulus supplied with $2 \mathrm{~V}$ attains stable temperature after less than 3 minutes so it will not elongate the preparation procedure and it gives opportunity to compare images from different readings of the same patient as well as different patients.

The biggest advantage of the proposed measurement device is that it is compact, the measurement is made at distance between 0,5 to $1 \mathrm{~m}$ from the patient and it does not have any special requirement to operate, so it is possible to use it even in a small consulting room which meets the criteria listed at point 2.2 .

Standard patch testing is performed as follows: strips of tape containing small quantities of common allergens are applied onto the skin, usually on the back or on the arm, and it must remain in place and be kept dry for 48 hours. At this time the surface of application should not be rubbed by, for example, an underwear or be scratched in case of false positive reactions. The patient should not take any cortisone medication during the test and avoid exposure of the back to the sun. After that time patches are removed and after several minutes initial reading is performed. After another 2 days final reading is made. Evaluation of test results is based on visual scheme. It is proceeded by physician on the basis of visual observation. Positive reaction at initial reading is classified as irritant contact dermatitis, while at further readings positive reaction is classified as ACD.

With usage of thermal medical imaging, before reading performed by physician, the patient has to acclimatize at consulting room with uncovered areas which are to be examined. Then the sequence of images is registered and after geometrical calibration obtained images will be overlapped for more precise analysis in ThermoAnalysis software (MONIT SHM), among other things with use of Pulsed Phase Thermography (PPT) to precise recover the gradient distribution of the thermal changes. Next stage is comparison of images from each readings and classification of lesions. Results obtained after that process will be compared with physician evaluation and saved to the data base. At Fig. 4 the procedure of IR thermography medical imaging acquisition and analysis is presented.

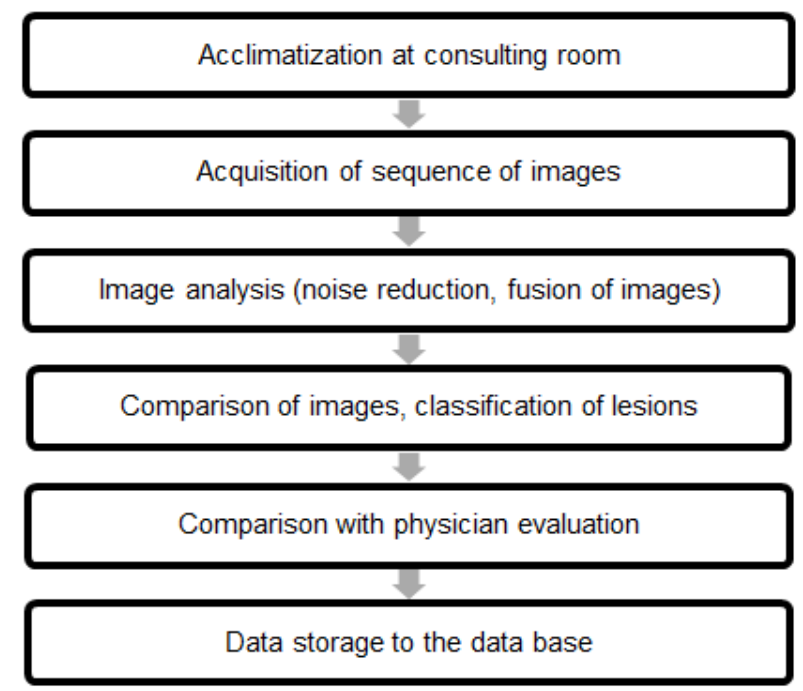

Fig. 4. The procedure of IR thermography medical imaging acquisition and analysis.

ThermoAnalysis is dedicated software designed to aid planning and carrying out measurements and thermal imaging tests, equipped with a module containing advanced tools for processing and analysing images to extract diagnostic features of infrared images. Operator may perform geometric analysis (time history plots, area observation), create 3D representation of a structure and use linear and non-linear processing functions. Advanced post-processing functions enable to work with single images or a group of images at the same time. Medical diagnostics is controlled and grouped in the projects with the possibility to create IR processing timestamp which allows to create different sections with subsequent processing techniques and parameters, thus provide to plan many diagnostic scenarios for acquired IR data and lead to compare various processing algorithms (Fig. 5). 


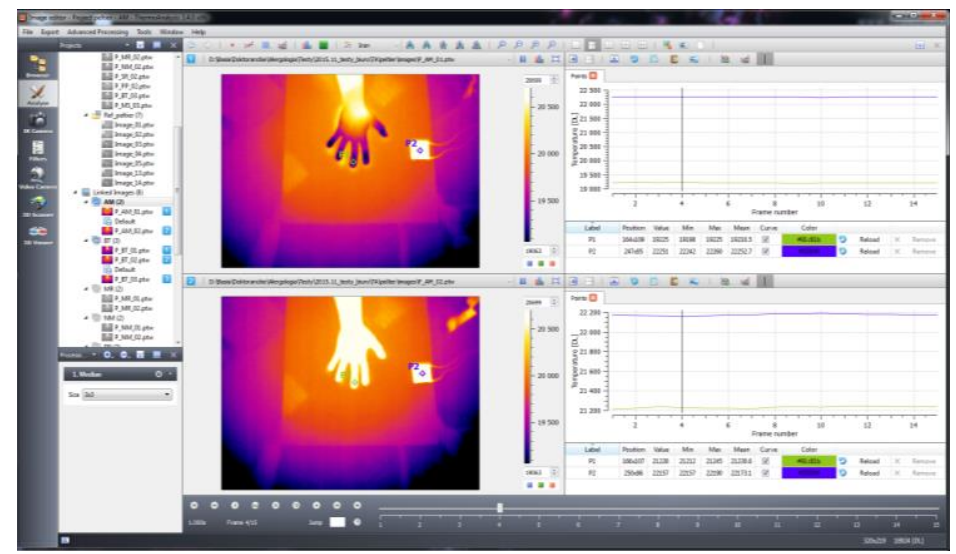

Fig. 5. Screen of the module for image analysis.

\section{Preliminary results}

To validate our method we performed initial tests with reference temperature module on hands of 7 healthy people. The IR camera (FLIR A300) had frame rate set at $3 \mathrm{~Hz}$, ambient temperature was $22^{\circ} \mathrm{C}$. Two sequences of images were captured: the first one after cooling, and the second one after 2 hours interval.

a)

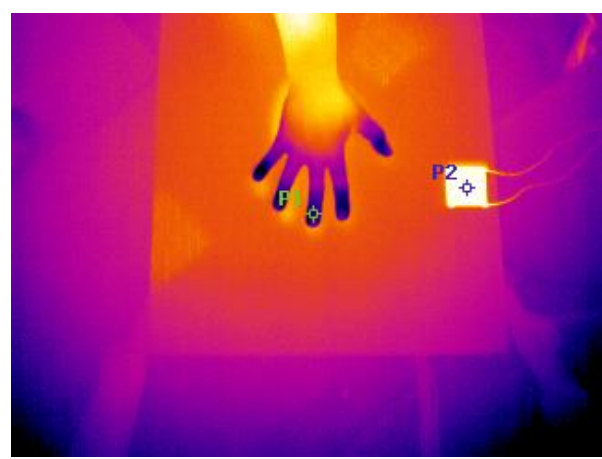

b)

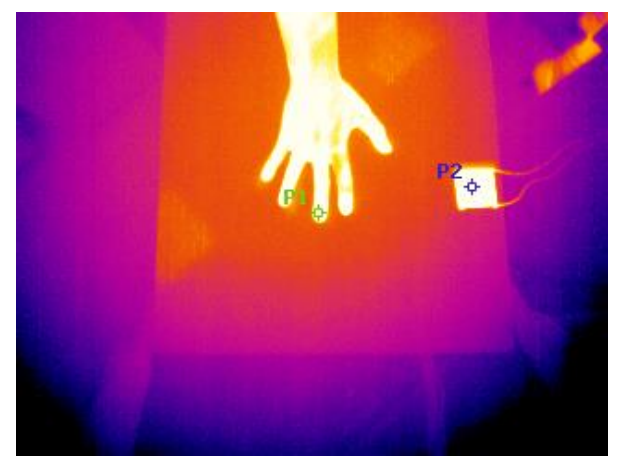

c)

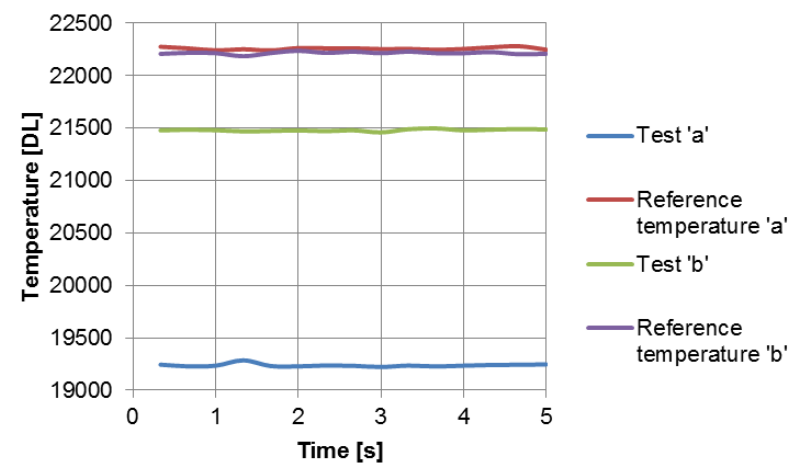

Fig. 6. Temperature measurement at point $P 1$ with use of reference temperature $P 2$ : a) image after cooling, b) image after 2 hours since cooling, c) temperature comparison.

Results presented at Fig. 6 shows that usage of reference temperature module makes possible to compare images acquired at different time by proper camera calibration and unification of the values of the histograms. For tests performed on patients it allows to check the difference in skin temperature at regions where patch tests were incubated in comparison to the neighbouring skin regions and reference temperature. 


\subsection{1/qirt.2016.136}

\section{Conclusions}

The main conclusions from the performed evaluations are that described method is promising and with introduced changes in measurement procedure it will enhance the percent of properly diagnosed patients. Furthermore, usage of our measurement unit gives opportunity to compare images with results from different readings of the same patient as well as different patients. The ability to view historical data can be helpful for physicians especially during evaluation of ambiguous cases.

For further research it is planned to create network-centric system to store the thermographs with physician description (diagnosis), case record, diagnostic results and description parameters. Those thermal images will be classified according to type of diagnosed pathology.

Medical thermal image processing is field of study which should be developed in the future, because it may significantly improve skin tests assessment. It can be a valuable additional method in allergology, however it cannot substitute standard visual evaluation of patch tests. Presented results are second stage of longer research process. Next step is to evaluate presented diagnostic procedure during standard patch tests performed on patients with usage of our measurement stand and advanced imaging analysis.

\section{REFERENCES}

[1] Laino L., di Carlo A., "Telethermography: an objective method for evaluating patch test reactions", Dermatology, vol. 20, No. 2, pp. 175-180, 2010.

[2] Dencheva M., Lyapina M., Kisselova A., et al., "Thermovision in dental allergology", Journal of IMAB, vol. 20, issue 3, pp. 558-562, 2014.

[3] ISO/TR 13154:2009 Medical electrical equipment-deployment, implementation and operational guidelines for identifying febrile humans using a screening thermograph

[4] IEC 80601-2-59:2008, Ed. 1.0 Medical electrical equipment-part 2-59: particular requirements for the basic safety and essential performance of screening thermography for human febrile temperature screening

[5] Diakides N.A., Bronzino J.D., ed., "Medical Infrared Imaging", CRC Taylor\&Francis, 2008.

[6] Ring E.F.J., Mcevoy H., Jung A., Zuber J., Machin G, "New standards for devices used for the measurement of human body temperature", Journal of Medical Engineering \& Technology, vol. 34, no. 4, pp. 249-253, 2010

[7] Streit M., Braathen L. R., "Contact dermatitis: clinics and pathology", Acta Odontol Scand, vol. 59, pp.309-314, 2001.

[8] Iris Ale S., Maibach H. I., „Irritant contact dermatitis versus allergic contact dermatitis”, Irritant Dermatitis, Chapter 2, pp. 11-18, 2006.

[9] http://www.icdrg.org (visited 23.04.2016)

[10] Fonacier L., Bernstein D., Pacheco K., et al., "Contact dermatitis: a practice parameter-Update 2015”, Journal of Allergy and Clinical Immunology: In Practice, vol. 3 no. 3S, 2015. (online access: https://www.aaaai.org/Aaaai/media/MediaLibrary/PDF\%20Documents/Practice\%20and\%20Parameters/Contact -dermatitis-2015.pdf)

[11] http://www.hopkinsmedicine.org/healthlibrary/conditions/dermatology/anatomy_of_the_skin_85,P01336/ (visited 20.12.2015)

[12] Chen M.M., Holmes K.R., "Microvascular contributions in tissue heat transfer", Ann. N.Y. Acad. Sci. 335, pp. 137-150, 1980.

[13] Pennes H.H., "Analysis of tissue and arterial blood temperatures in the resting human forearm", J. Appl. Physiol., 1:93-122, 1948.

[14] Lahiri B.B., Bagavathiappan S., Jayakumar, Philip J., "Medical applications of infrared thermography: A review", Infrared Physics \& Technology, vol. 55, pp. 221-235, 2012.

[15] Laino L., di Carlo A., "Telethermography: an objective method for evaluating patch test reactions", Dermatology, vol. 20, No. 2, pp. 175-180, 2010.

[16] Szentkuti A., Kavanagh H.S., Grazio S., "Infrared thermography and image analysis for biomedical use", Periodicum biologorum, vol. 113, no 4, pp. 385-392, 2011.

[17] Ring E.F.J., Ammer K., "Infrared thermal imaging in medicine", Physiological Measurement, vol. 33, pp. R33R46, 2012

[18] Szwedo M., Tomaka B., "Evaluation of patch tests results - research and development of technique based on infrared thermography", Diagnostyka, ISSN 1641-6414, vol. 16 no. 3, pp. 3-8, 2015. 